\title{
Paxillin is an intrinsic negative regulator of platelet activation in mice
}

\author{
Asuka Sakata ${ }^{1,2}$, Tsukasa Ohmori $^{{ }^{*}}$, Satoshi Nishimura ${ }^{1,3,4}$, Hidenori Suzuki ${ }^{5}$, Seiji Madoiwa ${ }^{1}$, Jun Mimuro ${ }^{1}$, \\ Kazuomi Kario ${ }^{2}$ and Yoichi Sakata ${ }^{1}$
}

\begin{abstract}
Background: Paxillin is a LIM domain protein localized at integrin-mediated focal adhesions. Although paxillin is thought to modulate the functions of integrins, little is known about the contribution of paxillin to signaling pathways in platelets. Here, we studied the role of paxillin in platelet activation in vitro and in vivo.

Methods and results: We generated paxillin knockdown (Pxn-KD) platelets in mice by transplanting bone marrow cells transduced with a lentiviral vector carrying a short hairpin RNA sequence, and confirmed that paxillin expression was significantly reduced in platelets derived from the transduced cells. Pxn-KD platelets showed a slight increased in size and augmented integrin allb $\beta 3$ activation following stimulation of multiple receptors including glycoprotein $\mathrm{VI}$ and $\mathrm{G}$ protein-coupled receptors. Thromboxane $\mathrm{A}_{2}$ biosynthesis and the release of a-granules and dense granules in response to agonist stimulation were also enhanced in Pxn-KD platelets. However, Pxn-KD did not increase tyrosine phosphorylation or intracellular calcium mobilization. Intravital imaging confirmed that Pxn-KD enhanced thrombus formation in vivo.
\end{abstract}

Conclusions: Our findings suggest that paxillin negatively regulates several common platelet signaling pathways, resulting in the activation of integrin allbß33 and release reactions.

Keywords: Platelet, Glycoprotein, Platelet aggregation, Release reaction

\section{Background}

A breakdown of normal platelet function results in either unexpected bleeding or thrombotic events [1]. Platelets are inactive in the intact vasculature under physiological conditions. However, once the platelets encounter an injured region of the endothelium, they attach through an interaction between von Willebrand factor and the glycoprotein (GP) Ib/IX/V complex [2], and then collagen receptor GPVI triggers platelet activation. Activated platelets release several classes of agonists, including ADP and thromboxane $(\mathrm{Tx}) \mathrm{A}_{2}$, which promote further platelet activation [3]. These steps ultimately increase the affinity of integrin $\alpha \operatorname{IIb} \beta 3$ for its ligands and induce platelet aggregation [4]. The intracellular signaling that increases the affinity of integrins is known as inside-out signaling [4]. Multiple signal transduction pathways from various

\footnotetext{
* Correspondence: tohmori@jichi.ac.jp

'Research Division of Cell and Molecular Medicine, Center for Molecular Medicine, Jichi Medical University School of Medicine, 3111-1 Yakushiji, Shimotsuke, Tochigi 329-0498, Japan

Full list of author information is available at the end of the article
}

receptors share common inside-out signaling cascades. For example, phosphoinositol hydrolysis, which leads to calcium mobilization and protein kinase $\mathrm{C}$ activation [5], and Rap1b activation are well-known signaling pathways that regulate integrin-mediated platelet functions [6].

To increase the affinity of integrin $\alpha \operatorname{IIb} \beta 3$, inside-out signaling pathways induce a drastic conformational change of the integrin [7]. Direct interactions between cytoskeletal proteins (e.g., talin and kindlin) and cytoplasmic $\beta$ integrin are essential for inducing the conformational change of integrins [7]. Indeed, the loss of talin or kindlin in platelets dramatically reduces integrin $\alpha \operatorname{IIb} \beta 3$-mediated platelet aggregation, despite normal expression levels of the surface receptors [8,9]. Selective blockade of talin binding by a single amino acid substitution in $\beta 3$ integrin also impairs integrin $\alpha \mathrm{IIb} \beta 3$-dependent platelet responses [10]. Although a number of integrin-associated proteins have been reported [11], the identities of proteins and their roles in regulating integrin signaling in platelets have not been fully characterized. It is also unknown whether 
additional molecules, other than talin and kindlin, are capable of regulating integrin signaling pathways.

Paxillin is a LIM domain protein that was originally identified as a substrate for oncogene $v$-src [12]. Paxillin contains two conserved structural domains, the $\mathrm{N}$-terminus and C-terminus, which consist of four LIM domains $[13,14]$. Two other family members have also been identified, Hic-5 and leupaxin [13,14]. Paxillin is ubiquitously expressed alongside these variants [13,14], except in human platelets that predominantly express Hic-5 $[15,16]$. Conversely, mouse platelets express paxillin and leupaxin in addition to Hic-5 [17]. Considering the multiple interaction motifs located within its structure, paxillin appears to serve as a signaling platform for the recruitment of numerous regulatory proteins near integrins $[13,14]$. Paxillin directly interacts with the cytoplasmic domain of integrin $\alpha 4$ and $\alpha 9$, but not $\alpha \mathrm{IIb}$, and these interactions controls integrin-mediated cell migration and spreading $[18,19]$.

Integrin $\alpha \operatorname{IIb} \beta 3$ in platelets is suitable for studies of integrin receptors because its ligand binding and signal transduction pathways are well characterized. Elucidating the intracellular proteins involved in the activation of integrin $\alpha I I b \beta 3$ can provide a better understanding of the functions of integrins and might result in the discovery of new antithrombotic targets [20]. We previously reported that lentiviral vector-mediated short hairpin RNA (shRNA) expression in hematopoietic stem cells greatly reduces the expression of the target protein in platelets [21]. This method enables functional analyses of target proteins that modulate platelet activation in anucleate platelets [21]. In the present study, we used this method to investigate the roles of paxillin in platelet activation, and found that paxillin negatively regulates platelet signaling pathways including the activation of integrin $\alpha \operatorname{IIb} \beta 3$ and release reactions.

\section{Materials and methods Materials}

All mouse cytokines were purchased from PeproTech (London, UK). The following antibodies and agonists were obtained from the specified suppliers: PAC-1 monoclonal antibody (mAb), anti-mouse P-selectin mAb (RB40.34), anti-paxillin mAb (clone 349), and anti-Hic-5 mAb (BD Biosciences, San Jose, CA); horseradish peroxidaseconjugated anti-green fluorescent protein (GFP) polyclonal antibody (Acris Antibodies, Himmelreich, Germany); phycoerythrin (PE)-Cy7-conjugated anti-mouse IgM (eBioscience, San Diego, CA); anti-talin mAb (clone 8D4); antiphosphotyrosine mAb (clone 4G10), and BAPTA-AM (Millipore, Billerica MA); human fibrinogen and epinephrine (Sigma-Aldrich, St. Louis, MO); anti-vinculin mAb (V284) (Chemicon, Billerica, MA); anti-mouse GPVI mAb (Six.E10), anti-mouse GPIb $\alpha$ mAb (Xia.G5), and anti-mouse integrin $\alpha \mathrm{IIb} \beta 3 \mathrm{mAb}$ (Leo.D2 and clone
JON/A) (Emfret Analytics, Eibelstadt, Germany); anti- $\alpha$ actin mAb (D6F6), anti-FAK polyclonal antibody, and anti-Src mAb (32G6) (Cell Signaling Technology, Danvers, MA); anti-Rap1b polyclonal antibody and anti-protein kinase $\mathrm{C} \alpha \mathrm{mAb}(\mathrm{M} 4)$ (Upstate Cell Signaling Solutions, Lake Placid, NY); allophycocyanin (APC)-conjugated antirat IgG polyclonal antibody (R\& D Systems, Minneapolis, $\mathrm{MN}$ ); convulxin (ALEXIS Biochemicals, Plymouth Meeting, PA); AYPGKF (Invitrogen, Carlsbad, CA); ADP (MC medical, Tokyo, Japan); U46619 (Cayman Chemical, Ann Arbor, MI).

\section{Lentiviral vector and virus production}

A lentiviral vector plasmid for expression of shRNA sequences and GFP (LentiLox vector) was purchased from the American Type Culture Collection (Manassas, VA) [22]. To efficiently express GFP in platelets, the cytomegalovirus promoter of the LentiLox vectors was substituted with the platelet-specific GPIb $\alpha$ promoter (LentiLox-GPIb $\alpha$ ) [21]. Putative shRNA sequences were designed using web-based software provided by Thermo Scientific Molecular Biology (http://www.thermoscientificbio.com/design-center/). Three shRNA sequences were synthesized for mouse paxillin and then cloned into a LentiLox vector plasmid (Additional files 1 and 2). Lentiviruses were produced as described previously [23].

\section{Transplantation of mouse bone marrow cells}

All animal procedures were approved by the Institutional Animal Care and Concern Committee of Jichi Medical University, and animal care was performed in accordance with the committee's guidelines. Mouse bone marrow cells (C57BL/6 J) were isolated and resuspended in StemPro ${ }^{\circ}-34$ SFM medium (Invitrogen) supplemented with $100 \mathrm{ng} / \mathrm{mL}$ each of stem cell factor, thrombopoietin, interleukin-6, and fms-like tyrosine kinase 3 ligand, and $200 \mathrm{ng} / \mathrm{mL}$ soluble interleukin-6 receptor. The lentiviral vector was added at $12-16 \mathrm{~h}$ after cell isolation (multiplicity of infection $[\mathrm{MOI}]=5)$, and the cell culture was continued for 21-22 h. Each recipient mouse (8-12 weeks of age) was irradiated with a single lethal dose of 9.5 Gy and then intravenously injected with $2 \times 10^{6}$ lentivirus-transduced bone marrow cells. After transplantation, about $50 \%$ of platelets expressed GFP (Figure 1). Mice with $70 \%$ of their platelets exhibiting GFP positivity were used in experiments that could not distinguish GFP-positive platelets, i.e., light transmission aggregometry, clot retraction, release concentration, calcium mobilization, and intravital microscopy.

\section{Immunoblotting}

Immunoblotting with the specific antibodies was performed as described previously [21]. To assess protein tyrosine phosphorylation, washed platelets were pretreated 


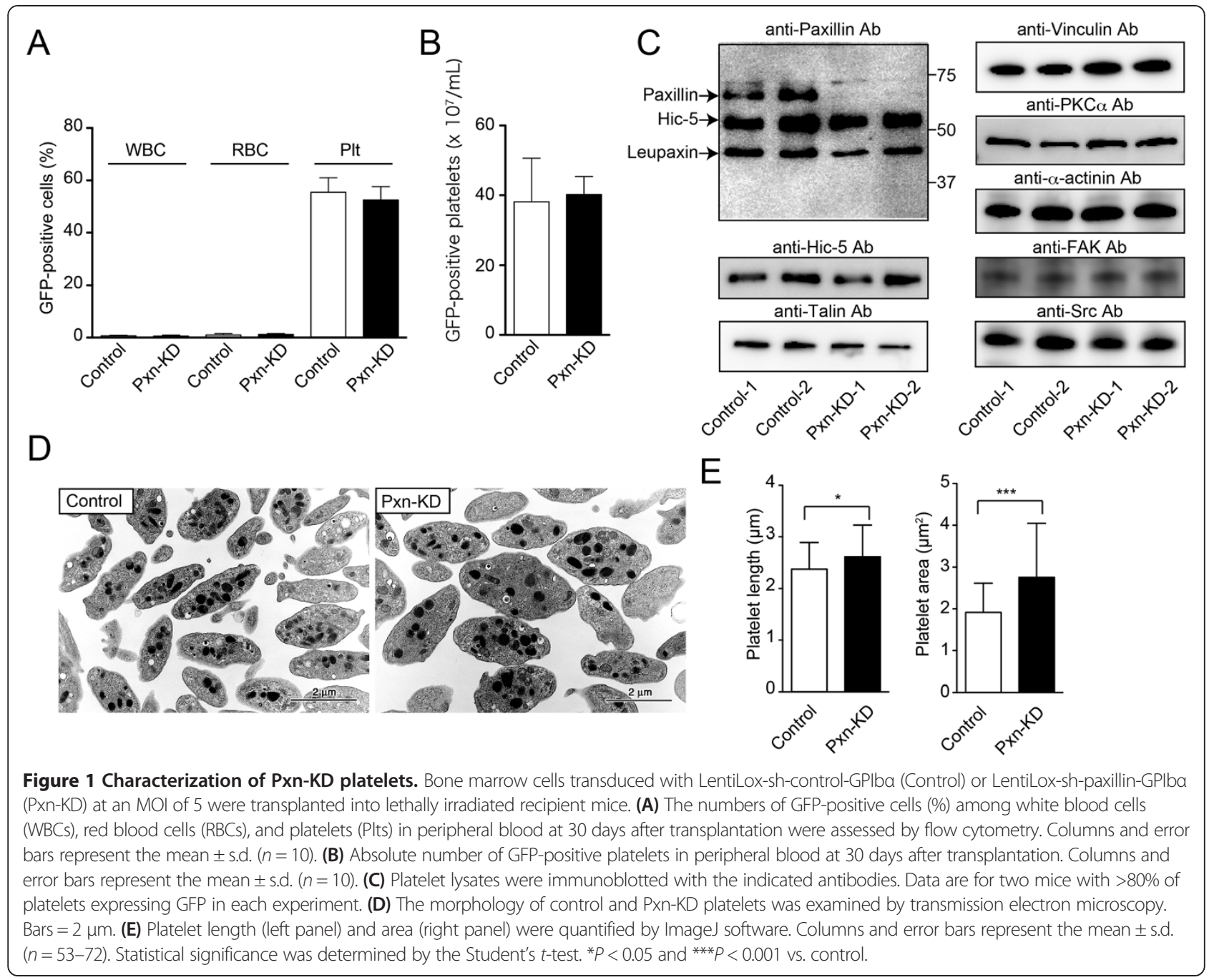

with $1 \mathrm{mmol} / \mathrm{L}$ EDTA, $5 \mathrm{U} / \mathrm{mL}$ apyrase, and $10 \mu \mathrm{mol} / \mathrm{L}$ SQ29548 to exclude the effects of aggregation, released $\mathrm{ADP}$, and $\mathrm{TxA}_{2}$.

\section{Transmission electron microscopy}

Mouse platelet pellets were fixed in 2\% glutaraldehyde in $0.1 \mathrm{~mol} / \mathrm{L}$ phosphate buffer $(\mathrm{pH} 7.4)$ for $60 \mathrm{~min}$ at $4^{\circ} \mathrm{C}$. The samples were washed, post-fixed with $1 \%$ osmium tetroxide in $0.1 \mathrm{~mol} / \mathrm{L}$ phosphate buffer for $60 \mathrm{~min}$ at $4^{\circ} \mathrm{C}$, dehydrated with a graded ethanol series, and then embedded in Epon (TAAB Laboratories, Aldermaston, UK) as described previously [24]. Ultrathin sections were prepared, stained with uranyl acetate and lead citrate, and then examined under a JEM1010 transmission electron microscope (JEOL, Tokyo, Japan) at an accelerating voltage of $80 \mathrm{kV}$. The length and area of platelets were quantified using ImageJ Ver. 10.2 for Macintosh (NIH, Bethesda, MD).

\section{Preparation of washed mouse platelets and flow} cytometry

A blood sample (100-400 $\mu \mathrm{L})$ was drawn from each mouse through the right jugular vein using a $30 \mathrm{G}$ syringe containing 1/10 sodium citrate, and then diluted with $3 \mathrm{~mL}$ Hepes/Tyrode buffer $(138 \mathrm{mmol} / \mathrm{L} \mathrm{NaCl}, 3.3 \mathrm{mmol} / \mathrm{L}$ $\mathrm{NaH}_{2} \mathrm{PO}_{4}, 2.9 \mathrm{mmol} / \mathrm{L} \mathrm{KCl}, 1 \mathrm{mmol} / \mathrm{L} \mathrm{MgCl}_{2}, 1 \mathrm{mg} / \mathrm{mL}$ glucose, and $20 \mathrm{mmol} / \mathrm{L}$ Hepes, $\mathrm{pH}$ 7.4). The diluted blood was centrifuged at $120 \times g$ for $8 \mathrm{~min}$, and the platelets obtained from the platelet-rich fraction were washed and resuspended in Hepes/Tyrode buffer. Just prior to centrifugation, a $15 \%$ acid-citrate-dextrose A solution and $0.1 \mu \mathrm{mol} / \mathrm{L}$ prostaglandin $\mathrm{I}_{2}$ were added to inhibit platelet activation. The final platelet suspensions were adjusted to $1 \times 10^{7}$ platelets $/ \mathrm{mL}$ and supplemented with $1 \mathrm{mmol} / \mathrm{L}$ $\mathrm{CaCl}_{2}$. To assess the binding of JON/A, a monoclonal antibody $(\mathrm{mAb})$ that recognizes activated mouse $\alpha \operatorname{IIb} \beta 3$ [25], to platelets, $30 \mu \mathrm{L}$ of washed platelets was incubated with $4 \mu \mathrm{L}$ of agonist solution, $4 \mu \mathrm{L}$ of phycoerythrin (PE)- 
conjugated JON/A and $1 \mu \mathrm{L}$ of biotin-conjugated antimouse P-selectin $\mathrm{mAb}$ for $5 \mathrm{~min}$, and then supplemented with $1 \mu \mathrm{L}$ of allophycocyanin (APC)-conjugated streptavidin. After $15 \mathrm{~min}$ of incubation, JON/A binding and P-selectin expression were determined by flow cytometry using a FACSAria Cell Sorter (Becton Dickinson, Mountain View, CA). Antibody binding was quantified as the mean fluorescence intensity (MFI) of GFP-positive platelets.

\section{Platelet aggregation}

Washed platelets were prepared as described above. The final suspensions were adjusted to $2 \times 10^{8}$ platelets $/ \mathrm{mL}$ and supplemented with $1 \mathrm{mmol} / \mathrm{L} \mathrm{CaCl}_{2}$ and $200 \mu \mathrm{g} / \mathrm{mL}$ fibrinogen. The aggregation response to agonist stimulation was measured based on light transmission measured using a PA-200 platelet aggregation analyzer (Kowa, Tokyo, Japan).

\section{Measurement of platelet products}

Washed platelets $\left(2 \times 10^{8} / \mathrm{mL}\right)$ were stimulated with the indicated agonists for $15 \mathrm{~min}$, and then the supernatants were recovered by centrifugation. The levels of platelet factor 4 (PF4) and serotonin in the supernatants were measured using a mouse PF4 enzyme-linked immunosorbent assay (ELISA) kit ( $\mathrm{R} \& \mathrm{D}$ Systems) and an anti-serotonin ELISA kit (GenWay Biotech, San Diego, CA), respectively. The levels of $\mathrm{TxB}_{2}$ in the supernatants were measured using an enzyme immunoassay (Cayman Chemical).

\section{Platelet adhesion}

Platelet adhesion to fibrinogen was assessed as described previously [21]. Briefly, eight-well dishes (Lab-Tek ${ }^{\oplus}$ Chamber Slide ${ }^{\mathrm{TM}}$ ) were coated with $400 \mu \mathrm{g} / \mathrm{mL}$ fibrinogen and then blocked with $1 \mathrm{mg} / \mathrm{mL}$ bovine serum albumin (BSA). Platelets were then added to the fibrinogen-coated dishes and incubated for $30 \mathrm{~min}$ at $37^{\circ} \mathrm{C}$. Adherent platelets were fixed with $3 \%$ paraformaldehyde and then permeabilized with phosphate-buffered saline (PBS) containing $0.3 \%$ Triton X-100 and 5\% donkey serum. After washing with $\mathrm{PBS}$, the platelets were incubated with an anti-GFP polyclonal antibody (MBL, Aichi, Japan). Bound antibodies were detected by Alexa Fluor 488-conjugated anti-rabbit IgG. Actin filaments were detected by staining with $1 \mu \mathrm{g} / \mathrm{mL}$ rhodamine-conjugated phalloidin. Immunofluorescence staining was observed and photographed under a confocal microscope (FV1000; Olympus, Tokyo, Japan). The spread area of GFP-positive platelets was quantified using ImageJ software. Because Pxn-KD platelets were slightly larger than control platelets (Figure 1), the mean platelet size determined by BSA staining was subtracted from the total area on fibrinogen to calculate the actual increase in platelet spreading.

\section{Clot retraction}

Human platelet-poor plasma was mixed with the same volume of Hepes/Tyrode buffer containing washed mouse platelets (final concentration: $3 \times 10^{8}$ platelets $/ \mathrm{ml}$ ). Plasma coagulation was initiated by addition of $0.1 \mathrm{U} / \mathrm{mL}$ thrombin. The clots were photographed at various time points after thrombin addition. When indicated, $0.5 \mathrm{mmol} / \mathrm{L}$ manganese was added to exclude the role of inside-out signaling. The two-dimensional area of serum formation extruded by clot retraction was quantified using ImageJ software and expressed as the progression of clot retraction.

\section{Calcium mobilization}

Platelets were incubated with GFP-Certified ${ }^{\mathrm{TM}}$ FluoForte $^{\mathrm{TM}}$ dye (Enzo Life Sciences, Farmingdale, NY). The fluorophore-loaded platelets $\left(2 \times 10^{8} / \mathrm{mL}\right)$ were resuspended in Hepes-Tyrode buffer containing $1 \mathrm{mmol} / \mathrm{L}$ EDTA, $5 \mathrm{U} / \mathrm{mL}$ apyrase, and $10 \mu \mathrm{mol} / \mathrm{L} \mathrm{SQ} 29548$ to exclude the effects of aggregation, extracellular calcium, released ADP, and $\mathrm{TxA}_{2}$. After stimulation, the intracellular calcium concentration was determined by monitoring the fluorescence (excitation, $530 \mathrm{~nm}$; emission, $570 \mathrm{~nm}$ ) using a microplate spectrofluorometer (Gemini EM; Molecular Devices, Sunnyvale, CA).

\section{Intravital microscopy and thrombus formation}

Intravital microscopy was performed to analyze thrombus formation in vivo as reported previously [26]. Briefly, Texas Red-dextran (100 mg/kg body weight [BW], molecular weight: $70 \mathrm{kDa}$; Invitrogen), Hoechst 33342 (10 mg/kg BW; Invitrogen), Dylight 488-conjugated anti-CD42b antibody (200 $\mu \mathrm{g} / \mathrm{kg} \mathrm{BW;} \mathrm{Emfret),} \mathrm{and} \mathrm{hematoporphyrin}(5 \mathrm{mg} / \mathrm{kg}$ BW; Sigma) were injected into anesthetized mice to produce reactive oxygen species (ROS) following laser irradiation. Blood cell dynamics were visualized during laser excitation (wavelengths 405, 488, and $561 \mathrm{~nm} ; 1.5 \mathrm{~mW}$ total power at $100 \times$ objective lens). After laser irradiation, sequential images of the mesentery were obtained using a resonance scanning confocal microscope (Nikon A1R; Nikon, Tokyo, Japan). The areas of thrombus (shown by antiCD42b antibody signals) before and after laser irradiation were calculated using NIS-Elements AR 3.2 (Nikon). When indicated, thrombus formation in the femoral artery was triggered by topical application of a filter paper tip saturated with $10 \% \mathrm{FeCl}_{3}$. After injection of Texas Red-dextran, Hoechst 33342, and Dylight 488-conjugated anti-CD42b antibody, thrombus formation was visualized and monitored by confocal microscopy using two photon microscopy (excitation wavelength $840 \mathrm{~nm}$ ) by NikonA1R MP (Nikon).

\section{Bleeding time}

The distal tail tip $(5 \mathrm{~mm})$ of an anesthetized mouse was clipped, and the tail was immediately immersed in PBS 
at $37^{\circ} \mathrm{C}$. Tail bleeding times were defined as the time required for the bleeding to stop.

\section{Results}

\section{Generation of paxillin knockdown (Pxn-KD) platelets}

To address the function of paxillin in mouse platelets, we used a lentiviral vector carrying shRNA sequences and GFP [22]. We synthesized three shRNA sequences for mouse paxillin, and cloned them into a LentiLox vector plasmid (Additional files 1 and 2). We selected one sequence that significantly inhibited paxillin expression in embryonic fibroblasts after transduction (Pxn-1 sequence; Additional files 1 and 2). After transplantation of bone marrow cells transduced with either the control or PxnKD sequence, about $50 \%$ of the platelets expressed GFP, and the absolute numbers of GFP-positive platelets did not differ between experiments using control and Pxn-KD sequences (Figure 1A-B). Furthermore, there was no effect on the total number of platelets (control: $6.8 \pm$ $1.72 \times 10^{8} / \mathrm{mL}$; Pxn-KD: $\left.7.7 \pm 0.65 \times 10^{8} / \mathrm{mL}, P=0.18\right)$. We compared the platelet aggregation response and release reaction in platelets from wild-type C57BL/6 J and control mice, and confirmed that platelet aggregation as well as the release reaction did not differ (data not shown). To confirm knockdown of paxillin in GFP-positive platelets, we selected mice in which more than $80 \%$ of platelets expressed GFP after transplantation. Immunoblotting of platelet lysates with an anti-paxillin mAb (clone 349) showed a marked reduction in paxillin expression following transplantation of bone marrow cells transduced with the Pxn-KD sequence (Figure $1 C$ ). This antibody also recognizes other members of the paxillin family, including Hic-5 and leupaxin [17]. However, Hic-5 and leupaxilin were not affected by expression of the Pxn-KD sequence (Figure 1C). Transmission electron microscopy of resting platelets revealed that the Pxn-KD platelets were slightly larger than control platelets (Figure 1D-E). This change was largely dependent on an increase of the cytoplasm volume, but not the granule volume (Additional file 3). Pxn-KD platelets showed marginally elevated expression levels of GPIb and integrin $\alpha \mathrm{IIb} \beta 3$, even though GPVI expression was normal (Additional file 4). These changes in Pxn-KD platelets were supposed to result from the increase in platelet size.

\section{Augmentation of integrin allb $\beta 3$ activation in Pxn-KD platelets}

We first focused on the role of paxillin in integrin $\alpha \operatorname{IIb} \beta 3$ activation that is critical for platelet aggregation. We performed flow cytometric analysis of integrin $\alpha \operatorname{IIb} \beta 3$ activation using an anti-JON/A mAb [25]. GFP-positive Pxn-KD platelets (Figure 2A, lower panel) showed significantly enhanced $\alpha \operatorname{IIb} \beta 3$ activation following stimulation compared with that of control platelets (Figure 2A, upper panel).
Enhanced JON/A binding of Pxn-KD platelets was observed following stimulation with the GPVI agonist convulxin and $G$ protein-coupled receptor agonists including a protease-activated receptor 4 agonist (AYPGKF), ADP, and U46619 (Figure 2A-B). However, JON/A binding was not enhanced in unstimulated or epinephrine-stimulated platelets, suggesting that Pxn-KD alone does not induce activation of integrin $\alpha \operatorname{IIb} \beta 3$. We next used light transmission aggregometry to assess platelet aggregation in vitro. We found that platelet aggregation was significantly augmented in Pxn-KD platelets, and this effect was evident at low agonist concentrations that induce platelet aggregation (Figure 2C-D).

\section{Enhanced release reactions and Tx biosynthesis in Pxn-KD platelets}

We next assessed the release reactions in response to stimulation. To address the role of paxillin in $\alpha$-granule secretion, P-selectin expression was determined in GFP-positive platelets by flow cytometry. As shown in Figure 3A-B, P-selectin expression in Pxn-KD platelets was significantly increased following stimulation with convulxin, AYPGKF, and U46619. In contrast, P-selectin expression was not increased by stimulation with ADP or epinephrine. We observed negligible increases in P-selectin expression of Pxn-KD platelets under the resting condition and after incubation with the fibronectin peptide Gly-Arg-Gly-Asp-Ser (GRGDS) (Figure 3B). To examine whether Pxn-KD platelets are already activated during circulation, we compared P-selectin expression in washed platelets and whole blood platelets before the preparation. An increase of P-selectin expression after washing the platelet preparation was observed in Pxn-KD platelets $(30.0 \pm 9.71$ to $37.2 \pm 5.72$ in the control vs. $27.8 \pm 2.56$ to $44.8 \pm 7.87, P<0.05)$, suggesting that the susceptibility of Pxn-KD platelets caused marginal activation during washing. Although PF4 and serotonin content in resting platelets did not differ between control and Pxn-KD platelets (Additional file 3), the actual release of PF4 and serotonin into the supernatant in response to platelet activation was also enhanced in Pxn-KD platelets (Figure 3C-D). Of note, a marked increase in $\mathrm{TxB}_{2}$ biosynthesis was observed in Pxn-KD platelets (Figure 3E). Pretreatment with the ADP scavenger apyrase and thromboxane $\mathrm{A}_{2}$ receptor antagonist SQ29548 somewhat corrected the increase of JON/A binding in Pxn-KD platelets. This result suggests that the extent of the increase of integrin activation is partially dependent on the release reaction (Additional file 5). Collectively, these data suggest that paxillin negatively regulates platelet activation signaling pathways leading to integrin activation, release reactions, and Tx synthesis. It is possible that general pathway (s) involved in platelet activation were enhanced by Pxn-KD, because platelet activation was increased in 


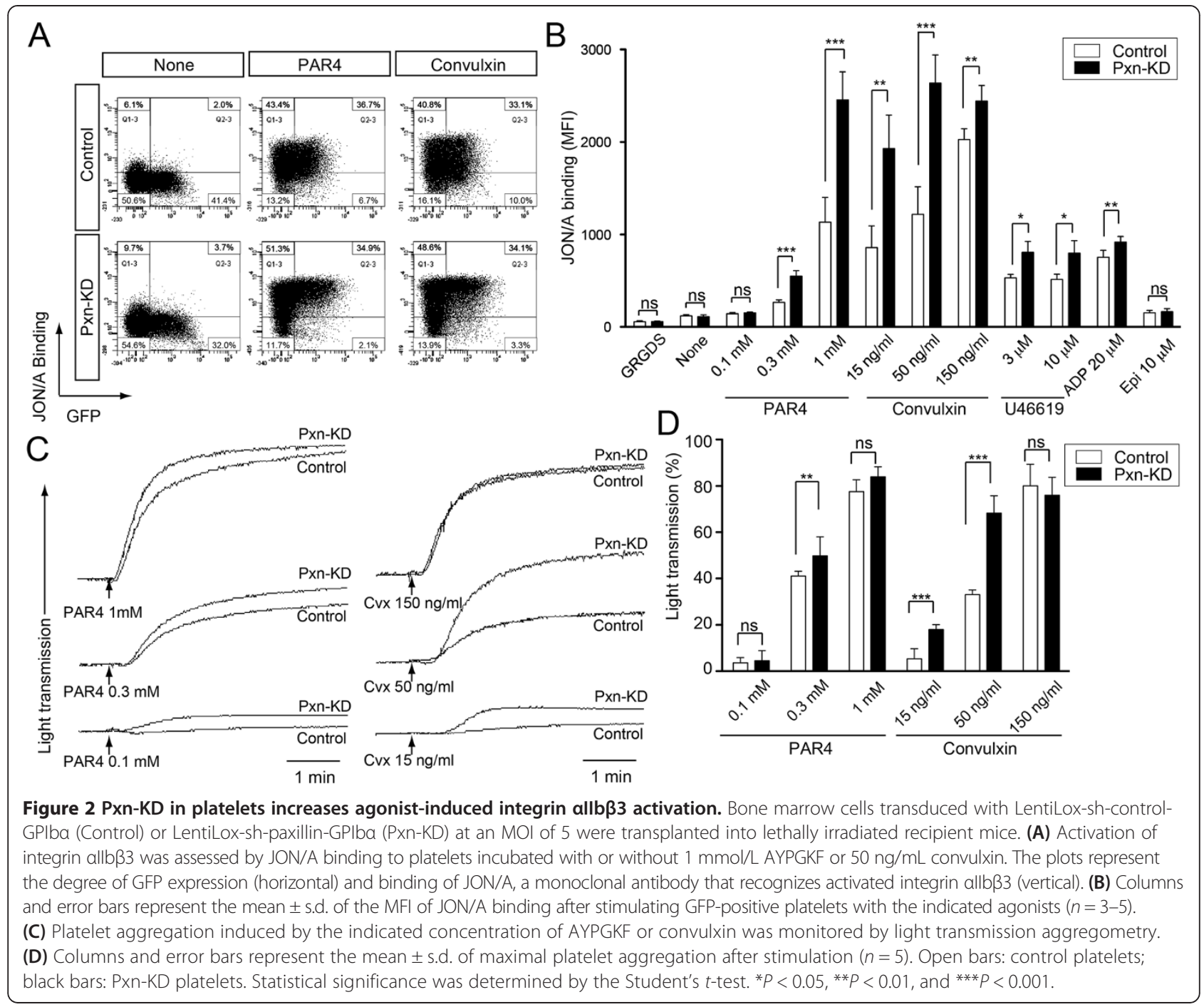

response to several classes of activators including GPVI and $\mathrm{G}$ protein-coupled receptors.

\section{Assessment of outside-in signaling pathways in Pxn-KD platelets}

To address the role of paxillin in outside-in signaling of integrin $\alpha \operatorname{Ilb} \beta 3$, we assessed platelet spreading on fibrinogen and clot retraction. The cell area independent of integrin outside-in signaling (i.e., adherent to the BSA control) was slightly increased in Pxn-KD platelets compared with that in control platelets (data not shown), because the Pxn-KD platelets were marginally larger than control platelets (Figure 1). To quantify the increase in platelet spreading, the mean platelet size on BSA was subtracted from the total spreading area on fibrinogen. As shown in Figure 4A, the increase in platelet spreading on fibrinogen without or with convulxin stimulation was significantly greater for Pxn-KD platelets than that for control platelets (Figure 4A-B). In addition, clot retraction induced by thrombin was significantly enhanced in PxnKD platelets compared with that in control platelets (Figure 4C-D). Acceleration of clot retraction in Pxn-KD platelets was also observed in the presence of manganese at $15 \mathrm{~min}(6.98 \pm 0.130$ vs. $7.56 \pm 0.072, P<0.05)$. These observations suggest that paxillin is an important regulator of integrin outside-in signaling via integrin $\alpha \operatorname{IIb} \beta 3$.

The role of paxillin in calcium mobilization in platelets

Because GPVI initiates signaling cascades by activation of non-receptor tyrosine kinases, we assessed tyrosine phosphorylation elicited by the GPVI signaling pathway. As a result, tyrosine phosphorylation events induced by convulxin were not affected by Pxn-KD (Figure 5A). The agonist-induced increase in intracellular calcium mobilization is an important common and proximal signaling event controlling platelet activation. Therefore, we next examined whether Pxn-KD enhanced intracellular calcium mobilization following stimulation. To exclude 


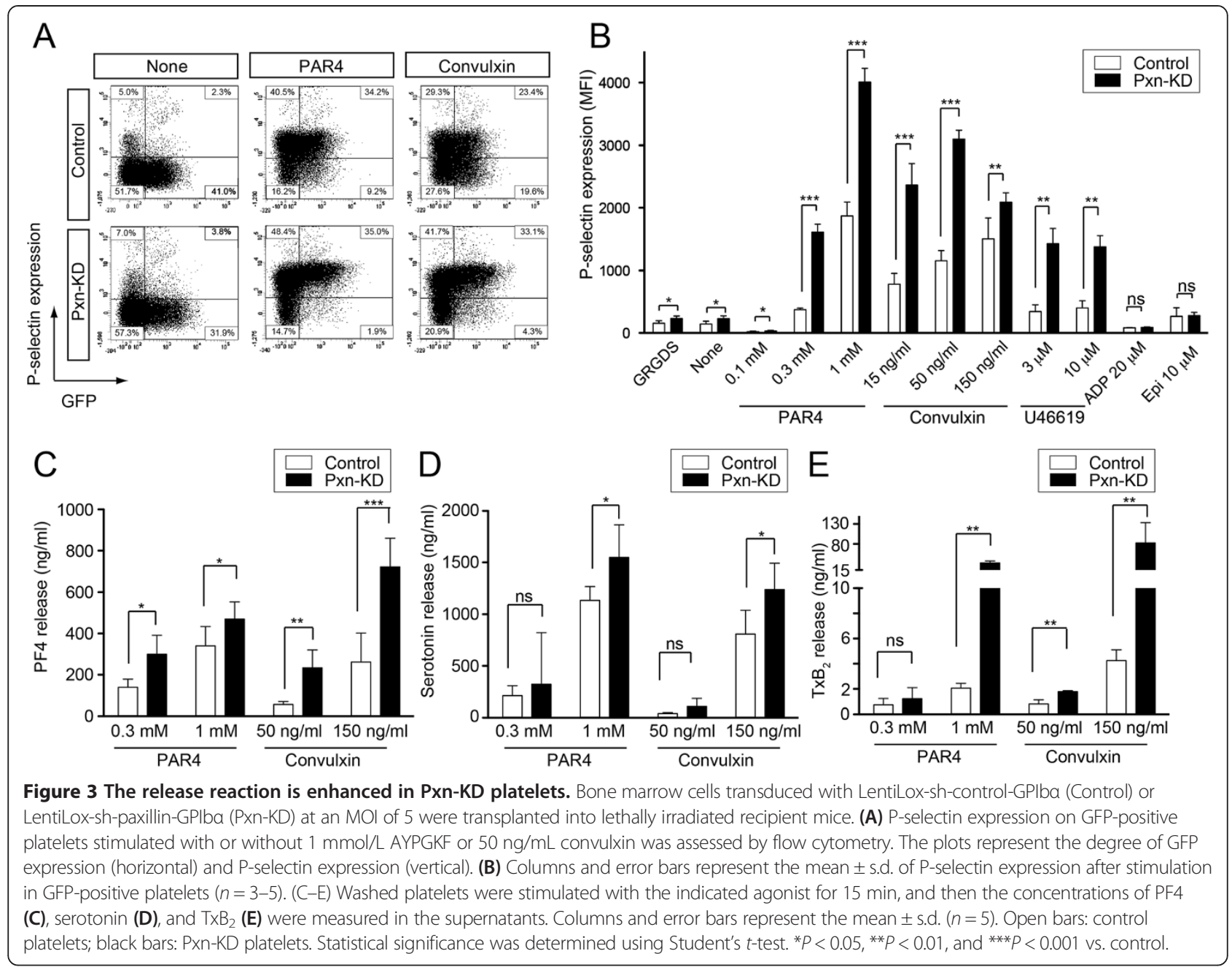

secondary effects of platelet aggregation, influx of extracellular calcium, and release reactions, we preincubated the platelets with EDTA, apyrase, and SQ29548. Intracellular calcium mobilization induced by the GPVI agonist convulxin and $G$ protein-coupled receptor stimulation with AYPGKF was rather decreased by Pxn-KD (Figure 5B). These data suggest that paxillin targets downstream signaling of calcium mobilization or a calcium-independent signaling pathway.

To explore the importance of calcium-independent signaling pathways in Pxn-KD platelets, we employed BAPTA-AM, an intracellular calcium chelator, to exclude the effect of calcium mobilization. Because JON/A requires extracellular calcium for antibody binding, we assessed P-selectin expression induced by an agonist. Pretreatment with BAPTA-AM significantly suppressed P-selectin expression in both control and Pxn-KD platelets (Figure $5 \mathrm{C}$ ). On the other hand, P-selectin expression elicited by an agonist was still observed in Pxn-KD platelets even in the presence of BAPTA-AM (Figure 5C).
These data indicate that downstream signaling from intracellular calcium mobilization is amplified by Pxn-KD, and the calcium-independent pathway is activated by Pxn-KD to increase platelet activation.

\section{Pxn-KD augments platelet adhesion and thrombus formation in vivo}

Finally, we examined the contribution of paxillin to thrombus formation in vivo. To visualize thrombus formation in vivo, we used a direct visual technique based on confocal microscopy in mesenteric capillaries [26]. Thrombus formation in this system was initiated by the production of ROS following laser irradiation [26]. Laser irradiation-induced thrombus formation was significantly enhanced in Pxn-KD platelets (Figure 6A and 6B and Additional files 6 and 7). In addition, there was an enhancement of thrombus formation initiated by $\mathrm{FeCl}_{3}$ in large femoral arteries (Additional file 8). Moreover, bleeding times after tail clipping significantly shortened in Pxn-KD experiments (Figure 6C). These findings support 


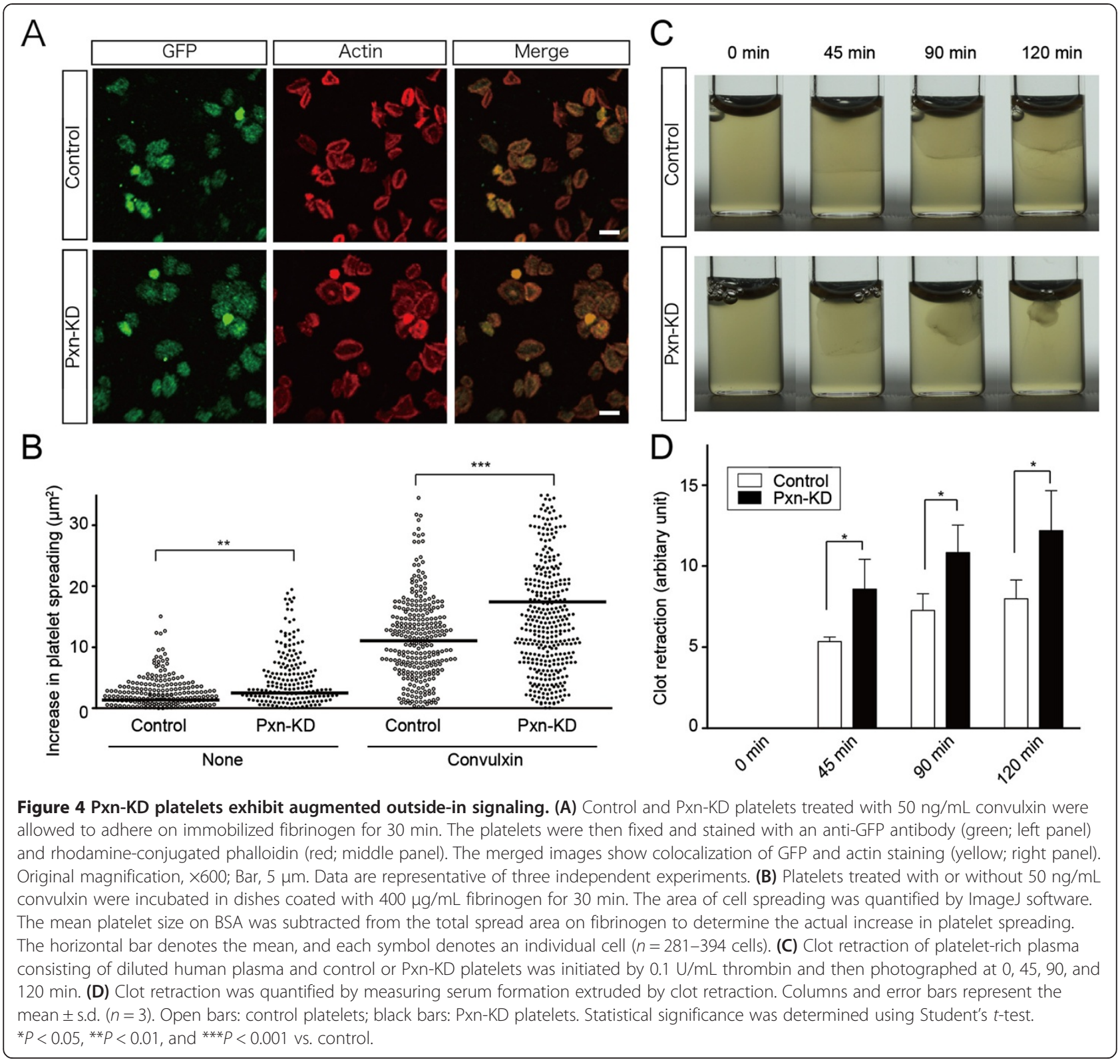

our hypothesis that paxillin is an important negative regulator of platelet activation and thrombus formation in vivo.

\section{Discussion}

Here, we found that the LIM protein paxillin is a negative regulator of platelet activation in mice. The negative regulation of platelet activation by paxillin was not limited to a specific signaling pathway, because Pxn-KD enhanced platelet activation in response to a variety of agonists. We also confirmed that thrombus formation was augmented in Pxn-KD platelets in vivo. This finding is notable because several previous reports suggest that changes in paxillin function actually reduce integrin signaling $[13,14]$. Furthermore, a previous finding in platelets has demonstrated the possible role of paxillin as a negative feedback regulator after integrin ligation to regulate the activity of Lyn tyrosine kinase [17]. However, this mode of regulation cannot fully explain the phenotypes of Pxn-KD platelets, because both outside-in and inside-out signaling were augmented by Pxn-KD. Our results reveal a new cellular function of paxillin and indicate new mechanisms that modulate platelet activation.

The most interesting result of this study was that PxnKD significantly enhanced the upstream signaling pathways that converge on platelet activation. Appropriate inhibition of the platelet response is essential to control pathological thrombus formation. It is well known that the mediators that enhance intracellular cAMP or cGMP 
A

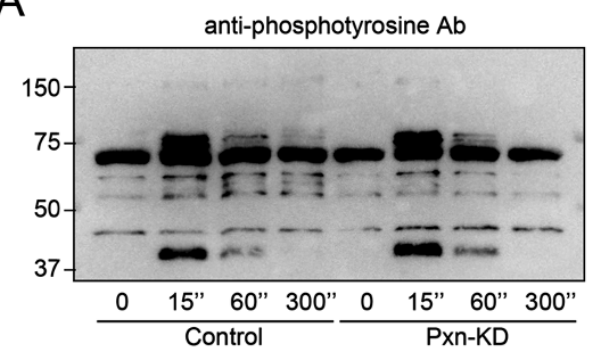

B

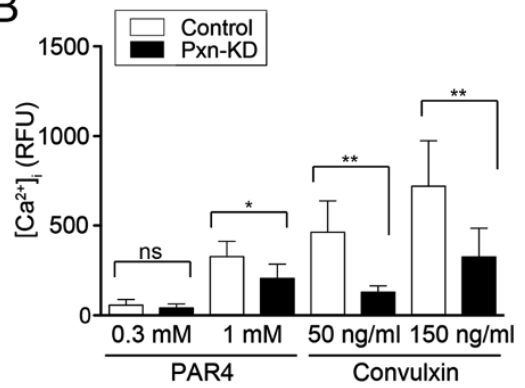

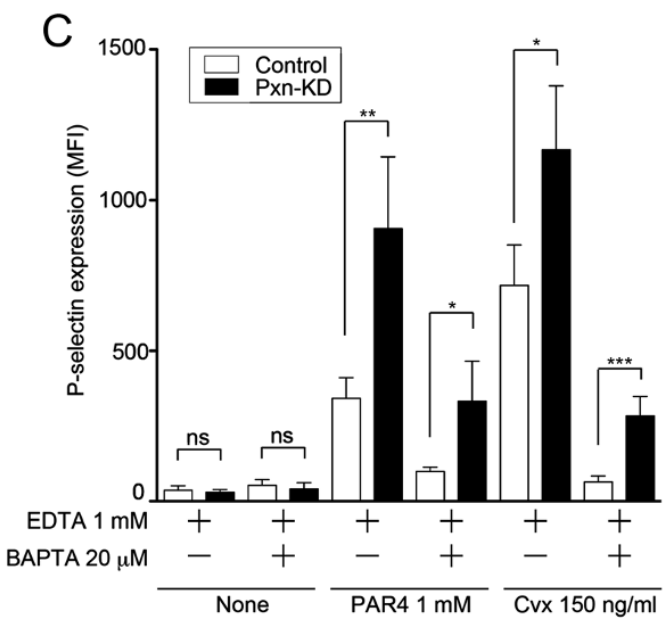

Figure 5 Pxn-KD fails to increase tyrosine phosphorylation and calcium mobilization. (A) Washed platelets obtained from control and Pxn-KD experiments were stimulated with $150 \mathrm{ng} / \mathrm{mL}$ convulxin for the indicated times. The cell lysates were resolved by SDS-polyacrylamide gel electrophoresis and then immunoblotted with an anti-phosphotyrosine mAb (4G10). The data shown are representative of three independent experiments. (B) Control and Pxn-KD platelets were labeled with GFP-Certified ${ }^{T M}$ FluoForte $^{T M}$ dye. Changes in intracellular calcium levels after stimulation with an indicated concentration of AYPGKF or convulxin were then measured every $30 \mathrm{~s}$. Data are expressed as the relative fluorescence unit (RFU) measured using a microplate spectrofluorometer (excitation, $530 \mathrm{~nm}$; emission, $570 \mathrm{~nm}$ ). The peak calcium concentration was measured after stimulation (open bars: control platelets; black bars: Pxn-KD platelets). Columns and error bars represent the mean \pm s.d. $(n=5-8)$. Statistical significance was determined by the Student's t-test. (C) Control and Pxn-KD platelets were pretreated with $1 \mathrm{mmol} / \mathrm{L}$ EDTA and/or 20 umol/L BAPTA-AM for $10 \mathrm{~min}$, and then stimulated with or without $1 \mathrm{mmol} / \mathrm{L}$ AYPGKF or $150 \mathrm{ng} / \mathrm{mL}$ convulxin. P-selectin expression on GFP-positive platelets was determined by flow cytometry. Columns and error bars represent the mean \pm s.d. of P-selectin expression $(n=4)$.

levels, including prostacyclin, prostaglandin $\mathrm{E}_{1}$, and nitric oxide, are strong extrinsic inhibitors of platelet activation [27]. These extrinsic mediators ameliorate the broad platelet activation elicited by various agonists [27]. Intrinsic negative regulators of platelet activation have been identified recently, but many of these proteins only control a specific receptor signaling pathway. GPVI-mediated immunoreceptor tyrosine-based activation motif (ITAM) signaling is regulated by immunotyrosine-based inhibitory motif (ITIM)-containing receptors including platelet endothelial cell adhesion molecule 1 and carcinoembryonic antigen-related cell adhesion molecule $1[28,29]$. Furthermore, Lyn tyrosine kinase has been reported to inhibit ITAM signaling by inducing tyrosine phosphorylation of ITIM [28]. It has also been reported that binding of a regulator of G-protein signaling to the Gio subunit limits platelet responsiveness to the receptor, which is independent of Rap1b [30]. Conversely, paxillin may downregulate platelet activity by modulating a common pathway, because Pxn-KD resulted in marked platelet hyperactivation in response to stimulation of tyrosine phosphorylation-based receptors and $\mathrm{G}$ protein-coupled receptors.

Although paxillin is reportedly involved in various integrin-mediated cellular functions, many of these functions are limited to outside-in signaling pathways. Paxillin-deficient embryos show embryonic lethality, and the phenotype closely resembles that of fibronectin-deficient mice [31]. Moreover, paxillin-deficient fibroblasts show reductions in cell migration and tyrosine phosphorylation following cell adhesion [31]. Chimeric integrin $\alpha \operatorname{IIb} \beta 3$ with a cytoplasmic tail substitution of $\alpha 4 \beta 1$ or $\alpha 9 \beta 1$, which facilitates paxillin binding, significantly inhibits cell spreading, but does not affect $\alpha \operatorname{IIb} \beta 3$-dependent cell adhesion $[18,19]$. Inhibition of paxillin binding to integrin $\alpha 4$ inhibits leukocyte recruitment to an inflammatory site [32]. These data suggest important roles of paxillin in outside-in signaling by direct interaction with the integrin $\alpha$-subunit. However, in this study, inside-out and outside- 

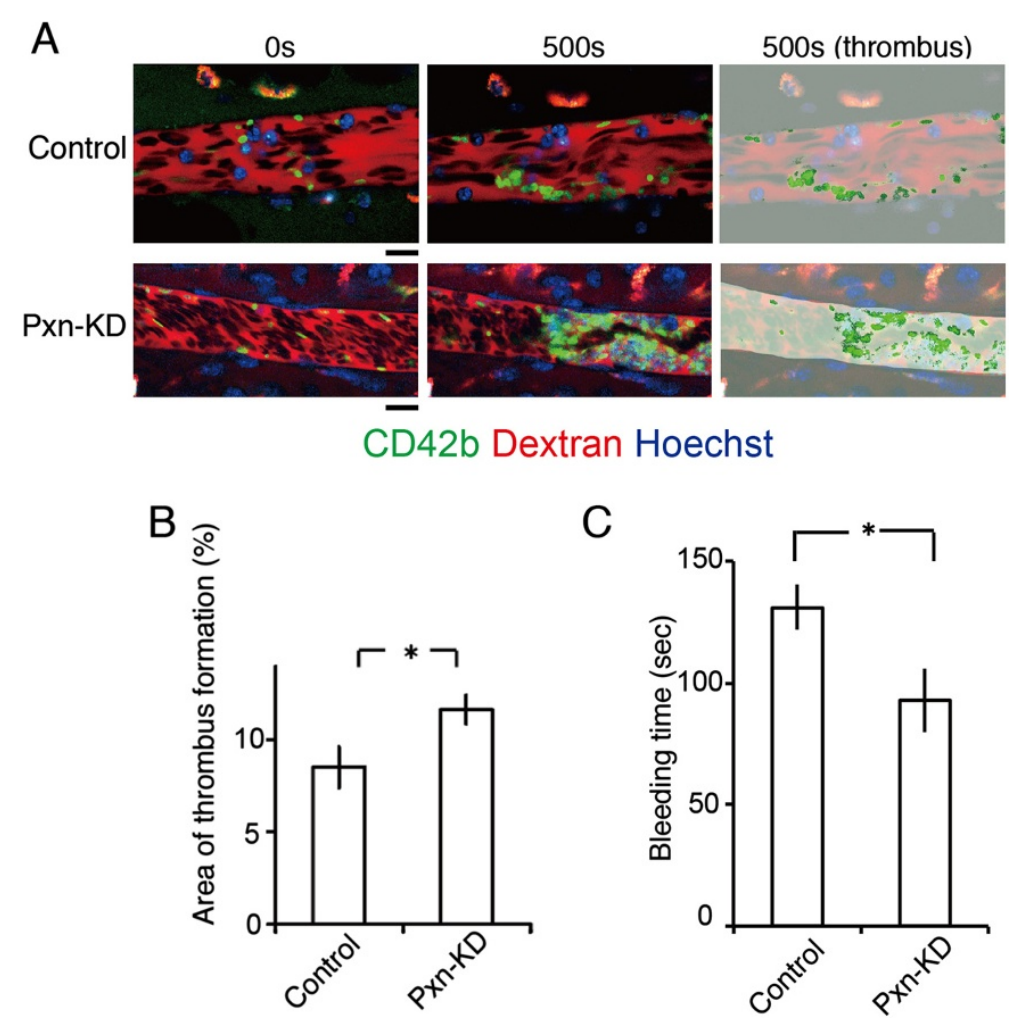

Figure 6 Pxn-KD in platelets expedites thrombus formation in vivo. (A) Intravital imaging of thrombus formation by laser irradiation of mesenteric arterioles in mice with control or Pxn-KD platelets. Thrombus formation was increased in mice with Pxn-KD platelets following laser irradiation. Bar, $10 \mu \mathrm{m}$. The right panel shows the results of quantification of the thrombus area. (B) Percentage areas of thrombus within blood vessels after laser irradiation. Columns and error bars represent the mean \pm s.e.m. ( $n=40$ vessels in five mice/group). (C) Tail bleeding times were assessed as described in the Methods. Columns and error bars represent the mean \pm s.e.m. ( $n=$ four mice/group). Statistical significance was determined using Student's t-test. ${ }^{*} P<0.05$ vs. control.

in signaling of integrin $\alpha \operatorname{IIb} \beta 3$ were increased in Pxn-KD platelets, even though paxillin failed to interact with platelet-specific integrin $\alpha$ IIb [19]. It is possible that other signaling pathways in platelets are modulated by paxillin, which is independent of direct interactions with integrins.

An issue that remains unresolved is the precise mechanism governing the negative regulatory function of paxillin in platelet activation. As described above, Rathore et al. previously reported that integrin $\alpha \operatorname{IIb} \beta 3$ dependent platelet aggregation induced tyrosine phosphorylation of paxillin and Hic-5 in platelets, leading to the binding of Csk, which controls activation of the Src family of tyrosine kinases [17]. Csk preferentially binds to paxillin in murine platelets that coexpress paxillin and Hic-5 [17]. Furthermore, the interaction abolishes the activity of Lyn, but not Fyn or Src. It is possible that paxillin acts as a negative feedback regulator of outsidein signaling by modulating Lyn activity after ligand binding to integrin $\alpha \operatorname{IIb} \beta 3$ [17]. However, this mechanism does not fully explain the functional roles of paxillin in platelets. Our data suggest that paxillin controls additional proximal signaling pathways for platelet activation.
Pxn-KD did not directly augment the conformational changes of integrin $\alpha \operatorname{Ilb} \beta 3$ expressed on Chinese hamster ovary cells (Additional file 9), tyrosine phosphorylation, or calcium mobilization induced by phosphoinositide turnover. These data suggest that paxillin negatively controls downstream signaling of calcium mobilization or a calcium-independent signaling pathway. In addition, calcium mobilization was rather reduced by Pxn-KD. It is therefore possible that negative feedback exists to prevent further activation of Pxn-KD platelets, or phosphoinositide turnover is directly modulated by Pxn-KD.

Our data suggest that several mechanisms may increase platelet activation by Pxn-KD. Notably, calcium-independent actions by Pxn-KD appear to exist, because P-selectin expression elicited by an agonist was still observed in Pxn-KD platelets even in the presence of BAPTA-AM. A previous report has suggested that coordinated signaling through both $G_{12 / 13}$ and $G_{i}$ causes integrin $\alpha I I b \beta 3$ activation, despite a small increase in intracellular calcium [33]. In addition, $G_{12 / 13}$ and $G_{i}$ signaling activates integrin $\alpha \mathrm{IIb} \beta 3$ in Gq-deficient mice [34]. It is possible that paxillin modify the calcium-independent signaling 
pathway leading to release reaction and integrin $\alpha \operatorname{IIb} \beta 3$ activation. Additional studies are needed to investigate how paxillin regulates platelet activation, and to assess whether these roles of paxillin in control of cellular signaling are common mechanisms in other cell types. We are now interested in further investigation of the precise mechanisms, and additional experiments are currently underway in our laboratory.

Another interesting finding of our study is that Pxn-KD resulted in an enlargement of platelet volume. CLP36, a member of the LIM domain family, was recently reported to play some roles in platelet activation [35]. Platelets from mice lacking the LIM domain of CLP36 show a slight increase in size and hyperactivation in response to a GPVI agonist [35]. The phenotypes of CLP36-deficient or mutant platelets are similar to those of Pxn-KD platelets in our study, although $\mathrm{G}$ protein-coupled receptor signaling is not affected in CLP36-deficient or mutant mice. Accordingly, the expression of LIM domain proteins may determine platelet size and reactivity.

To extrapolate the implications of our study to the biology and pathophysiology of humans, we must consider the differential expression pattern of paxillin-related proteins in platelets among species. Murine platelets express paxillin, Hic-5, and leupaxin, whereas human platelets only express Hic-5 [17]. Hagmann et al. reported that a switch from paxillin to Hic-5 expression should occur during the late phase of megakaryopoiesis in humans [15]. A recent report has described platelet functions in Hic-5deficient mice [36]. Hic-5-deficient mice exhibit prolonged bleeding times, and the loss of Hic-5 in platelets slightly impairs integrin $\alpha \operatorname{IIb} \beta 3$ activation induced by thrombin, but not other agonists including convulxin, U46619, and ADP [36]. Although the hemostatic defect in Hic-5deficient mice, as assessed by tail bleeding, is not fully explained by a mild defect in platelet function, it is possible that the structurally related proteins paxillin and Hic-5 play opposing roles in the regulation of platelet function in murine platelets. Leupaxin, another LIM protein that is predominantly expressed in leukocytes, has been reported to play an inhibitory role in B cell receptor signaling [37], which is similar to the role of paxillin reported in this study. In human platelets, which only express Hic-5, it will be necessary to elucidate whether Hic -5 acts as a positive regulator of integrin $\alpha \mathrm{IIb} \beta 3$ activation.

In summary, we have shown that paxillin is a negative regulator of platelet activation in mouse platelets. Modulation of platelet activation by Pxn-KD may originate in the augmentation of common signaling pathways, leading to integrin $\alpha \operatorname{IIb} \beta 3$ activation, release reactions, and Tx biosynthesis. Modulation of the LIM protein function might be an attractive candidate therapeutic target capable of strongly suppressing unexpected platelet activation in thrombotic disorders. The next challenge will be elucidating the precise mechanism by which paxillin regulates the signaling pathway in platelet activation.

\section{Additional files}

Additional file 1: Schematic diagrams of the lentiviral vector used in this study. (A) Schematic diagram of the lentiviral vector. (B) Locations of the oligonucleotides encoding the shRNAs in the mouse paxillin (Pxn) gene. (C) Mouse embryonic fibroblasts were transduced with a lentiviral vector containing the control, Pxn-1, Pxn-2, or Pxn-3 shRNA sequences at MOls of 1,3 , or 10 . Protein expression was determined by immunoblotting at $48 \mathrm{~h}$ after transduction. Data are representative of three independent experiments.

Additional file 2: Oligonucleotide sequences of siRNA cloned into LentiLox.

Additional file 3: Pxn-KD does not affect granule contents. Bone marrow cells transduced with LentiLox-sh-control-GPIba (Control) or LentiLox-sh-paxillin-GPIba (Pxn-KD) at an MOI of 5 were transplanted into lethally irradiated recipient mice. (A) The morphology of control and Pxn-KD platelets was examined by transmission electron microscopy, and the areas of granules and cytoplasm in each platelet were independently quantified using ImageJ software for Macintosh. Columns and error bars represent the mean \pm s.d. $(n=53-70)$. (B-C) Washed platelets were lysed to measure the concentrations of platelet factor 4 (PF4) (B) and serotonin (C). Columns and error bars represent the mean \pm s.d. $(n=4)$. Statistical significance was determined using Student's $t$ test. ${ }^{* * *} P<0.001$ vs. control.

Additional file 4: Expression levels of platelet-specific glycoproteins. Description of data: (A) Expression levels of GPIlb/Illa (integrin allb33) (left panel), GPIb (middle panel), and GPVI (right panel) in control (dark gray) and paxillin-knockdown platelets (light gray). (B) Columns and error bars represent the mean \pm s.d. of the mean fluorescence intensity (MFI) of antibody binding $(n=5)$. Statistical significance was determined using Student's $t$ test. ${ }^{*} P<0.05$, ${ }^{* *} P<0.01$, and ${ }^{* * *} P<0.001$ vs. control.

Additional file 5: Effects of apyrase and SQ29548 on agonist-induced integrin allb $\beta 3$ activation and P-selectin expression in control and Pxn-KD platelets. Platelets pretreated without or with $5 \mathrm{U} / \mathrm{mL}$ apyrase and $10 \mu \mathrm{mol} / \mathrm{L}$ SQ29548 were stimulated with the indicated agonists. JON/A binding (A) and P-selectin expression (B) on GFP-positive platelets were assessed by flow cytometry. Column and error bars represent the mean $\pm \mathrm{s}$. d. of the mean fluorescence intensity (MFI) $(n=3-4)$. Statistical significance was determined using Student's $t$ test. ${ }^{*} P<0.05$, ${ }^{* *} P<0.01$, and ${ }^{* *} P<0.001$ vs. control.

Additional file 6: Intravital imaging of thrombus formation by laser irradiation of mesenteric arterioles in mouse with control platelets.

Additional file 7: Intravital imaging of thrombus formation by laser irradiation of mesenteric arterioles in mice with Pxn-KD platelets.

Additional file 8: Thrombus formation in femoral arteries induced by $\mathrm{FeCl} 3$. (A) Intravital imaging of thrombus formation 5 mins after $\mathrm{FeCl} 3$ treatment in femoral arteries in mice with control or paxillin knock-down platelets (Pxn-KD). The black arrows indicate the direction of blood flow, and triangles show the developed thrombus. Bar, $100 \mu \mathrm{m}$. (B) Areas of thrombus within arteries 20 mins after laser irradiation. Columns and error bars represent the mean \pm s.e.m. ( $n=8$ arteries in four mice/group).

Additional file 9: Knock-down of paxillin does not affect talin-dependent activation of integrin allb $\beta 3$ in $\mathrm{CHO}$ cells. (A) Schematic representation of the lentiviral vectors used in this experiment. (B-D) allb $33-\mathrm{CHO}$ cells were transduced with lentiviral vectors expressing a control shRNA sequence and GFP (Control), the paxillin shRNA sequence and GFP (Pxn-KD), a control shRNA sequence and the GFP-Talin FERM domain (Control-FERM), or the paxillin shRNA sequence and the GFP-Talin FERM domain (Pxn-KD-FERM). (B) Lysates obtained from the transduced cells were immunoblotted with anti-GFP polyclonal antibody, anti-paxillin monoclonal antibody, and anti-vinculin monoclonal antibody. (C) PAC-1 binding after transduction in the presence or absence of $1 \mathrm{mmol} / \mathrm{L}$ GRGDS was assessed by 
flow cytometry. Data are representative of four independent experiments.

(D) Columns and error bars represent the mean \pm s.d. of PAC -1 binding $(n=4)$. Statistical significance was determined using Student's $t$ test.

\section{Abbreviations}

Pxn-KD: Paxillin-knockdown; Tx: Thromboxane; shRNA: Short hairpin RNA; GRGDS: Gly-Arg-Gly-Asp-Ser; ROS: Reactive oxygen species;

ITAM: Immunoreceptor tyrosine-based activation motif;

ITIM: Immunotyrosine-based inhibitory motif.

\section{Competing interests}

The authors declare that they have no competing interests.

\section{Authors' contributions}

Contribution: AS, TO, and SN designed the study, performed the experiments, analyzed the data, and wrote the manuscript; HS performed the experiments and wrote the manuscript; SM, JM, KK, and YS analyzed the data and revised the manuscript. All authors read and approved the final manuscript.

\section{Acknowledgements}

We would like to thank Tamaki Aoki, Naoko Ito and Masanori Ito (Jichi Medical University) for providing excellent technical assistance. This study was supported by Grants-in-Aid for Scientific Research (T.O., S.M., J.M.), the Funding Program for Next Generation World-Leading Researchers (S.N.), the Translational Systems Biology and Medicine Initiative from JST (S.N.), the Support Program for Strategic Research Infrastructure from the Japanese Ministry of Education and Science (Y.S.), and Health, Labour and Science Research Grants for Research on HIV/AIDS and Research on Intractable Diseases from the Japanese Ministry of Health, Labour and Welfare (Y.S.).

\section{Author details}

${ }^{1}$ Research Division of Cell and Molecular Medicine, Center for Molecular Medicine, Jichi Medical University School of Medicine, 3111-1 Yakushiji, Shimotsuke, Tochigi 329-0498, Japan. Division of Cardiovascular Medicine, Department of Internal Medicine, Jichi Medical University School of Medicine, 3111-1 Yakushiji, Shimotsuke, Tochigi 329-0498, Japan. ${ }^{3}$ Department of Cardiovascular Medicine, The University of Tokyo, 7-3-1 Hongo, Bunkyo, Tokyo 113-8655, Japan. ${ }^{4}$ Translational Systems Biology and Medicine Initiative, The University of Tokyo, 7-3-1 Hongo, Bunkyo, Tokyo 113-8655, Japan. ${ }^{5}$ Department of Morphological and Biomolecular Research, Nippon Medical School, 1-1-5 Sendagi, Bunkyo, Tokyo 113-8602, Japan.

Received: 26 November 2013 Accepted: 9 December 2013 Published: 2 January 2014

\section{References}

1. Coller BS: Historical perspective and future directions in platelet research. J Thromb Haemost 2011, 9(Suppl 1):374-395.

2. Watson SP, Auger JM, McCarty OJ, Pearce AC: GPVI and integrin alphallb beta3 signaling in platelets. J Thromb Haemost 2005, 3:1752-1762.

3. Varga-Szabo D, Pleines I, Nieswandt B: Cell adhesion mechanisms in platelets. Arterioscler Thromb Vasc Biol 2008, 28:403-412.

4. Shattil SJ, Kim C, Ginsberg MH: The final steps of integrin activation: the end game. Nat Rev Mol Cell Biol 2010, 11:288-300.

5. Siess W: Molecular mechanisms of platelet activation. Physiol Rev 1989, 69:58-178.

6. Guidetti GF, Torti M: The small GTPase rap1b: a bidirectional regulator of platelet adhesion receptors. J Signal Transduct 2012, 2012:412089.

7. Moser M, Legate $K R$, Zent $R$, Fassler R: The tail of integrins, talin, and kindlins. Science 2009, 324:895-899.

8. Moser M, Nieswandt B, Ussar S, Pozgajova M, Fassler R: Kindlin-3 is essential for integrin activation and platelet aggregation. Nat Med 2008, 14:325-330.

9. Nieswandt B, Moser M, Pleines I, Varga-Szabo D, Monkley S, Critchley D, Fassler R: Loss of talin1 in platelets abrogates integrin activation, platelet aggregation, and thrombus formation in vitro and in vivo. J Exp Med 2007, 204:3113-3118
10. Petrich BG, Fogelstrand P, Partridge AW, Yousefi N, Ablooglu AJ, Shattil SJ, Ginsberg MH: The antithrombotic potential of selective blockade of talin-dependent integrin alpha Ilb beta 3 (platelet GPIIb-IIla) activation. J Clin Invest 2007, 117:2250-2259.

11. Cantor JM, Ginsberg MH, Rose DM: Integrin-associated proteins as potential therapeutic targets. Immunol Rev 2008, 223:236-251.

12. Glenney JR Jr, Zokas L: Novel tyrosine kinase substrates from Rous sarcoma virus-transformed cells are present in the membrane skeleton. J Cell Biol 1989, 108:2401-2408.

13. Brown MC, Turner CE: Paxillin: adapting to change. Physiol Rev 2004, $84: 1315-1339$

14. Deakin NO, Turner CE: Paxillin comes of age. J Cell Sci 2008, 121:2435-2444.

15. Hagmann J, Grob M, Welman A, Van Willigen G, Burger MM: Recruitment of the LIM protein hic- 5 to focal contacts of human platelets. J Cell Sci 1998, 111(Pt 15):2181-2188.

16. Osada M, Ohmori T, Yatomi Y, Satoh K, Hosogaya S, Ozaki Y: Involvement of Hic-5 in platelet activation: integrin alphallbbeta3dependent tyrosine phosphorylation and association with proline-rich tyrosine kinase 2. Biochem J 2001, 355:691-697.

17. Rathore VB, Okada M, Newman PJ, Newman DK: Paxillin family members function as Csk-binding proteins that regulate Lyn activity in human and murine platelets. Biochem J 2007, 403:275-281.

18. Liu S, Slepak M, Ginsberg MH: Binding of Paxillin to the alpha 9 integrin cytoplasmic domain inhibits cell spreading. J Biol Chem 2001, 276:37086-37092.

19. Liu S, Thomas SM, Woodside DG, Rose DM, Kiosses WB, Pfaff M, Ginsberg $\mathrm{MH}$ : Binding of paxillin to alpha4 integrins modifies integrin-dependent biological responses. Nature 1999, 402:676-681.

20. Coller BS, Anderson K, Weisman HF: New antiplatelet agents: platelet GPIlb/Illa antagonists. Thromb Haemost 1995, 74:302-308.

21. Ohmori T, Kashiwakura Y, Ishiwata A, Madoiwa S, Mimuro J, Sakata Y: Silencing of a targeted protein in in vivo platelets using a lentiviral vector delivering short hairpin RNA sequence. Arterioscler Thromb Vasc Biol 2007, 27:2266-2272.

22. Rubinson DA, Dillon CP, Kwiatkowski AV, Sievers C, Yang L, Kopinja J, Rooney DL, Ihrig MM, McManus MT, Gertler FB, et al: A lentivirus-based system to functionally silence genes in primary mammalian cells, stem cells and transgenic mice by RNA interference. Nat Genet 2003, 33:401-406.

23. Ohmori T, Kashiwakura Y, Ishiwata A, Madoiwa S, Mimuro J, Furukawa Y, Sakata $Y$ : Vinculin is indispensable for repopulation by hematopoietic stem cells, independent of integrin function. J Biol Chem 2010, 285:31763-31773.

24. Suzuki H, Okamura Y, Ikeda Y, Takeoka S, Handa M: Ultrastructural analysis of thrombin-induced interaction between human platelets and liposomes carrying fibrinogen gamma-chain dodecapeptide as a synthetic platelet substitute. Thromb Res 2011, 128:552-559.

25. Bergmeier W, Schulte V, Brockhoff G, Bier U, Zirngibl H, Nieswandt B Flow cytometric detection of activated mouse integrin alphallbbeta 3 with a novel monoclonal antibody. Cytometry 2002, 48:80-86.

26. Nishimura S, Manabe I, Nagasaki M, Kakuta S, Iwakura Y, Takayama N, Ooehara J, Otsu M, Kamiya A, Petrich BG, et al: In vivo imaging visualizes discoid platelet aggregations without endothelium disruption and implicates contribution of inflammatory cytokine and integrin signaling. Blood 2012, 119:e45-e56.

27. Smolenski A: Novel roles of CAMP/cGMP-dependent signaling in platelets. J Thromb Haemost 2012, 10:167-176.

28. Ming Z, Hu Y, Xiang J, Polewski P, Newman PJ, Newman DK: Lyn and PECAM-1 function as interdependent inhibitors of platelet aggregation. Blood 2011, 117:3903-3906.

29. Wong C, Liu Y, Yip J, Chand R, Wee JL, Oates L, Nieswandt B, Reheman A, $\mathrm{Ni} \mathrm{H}$, Beauchemin N, Jackson DE: CEACAM1 negatively regulates platelet-collagen interactions and thrombus growth in vitro and in vivo. Blood 2009, 113:1818-1828.

30. Signarvic RS, Cierniewska A, Stalker TJ, Fong KP, Chatterjee MS, Hess PR, Ma P, Diamond SL, Neubig RR, Brass LF: RGS/Gi2alpha interactions modulate platelet accumulation and thrombus formation at sites of vascular injury. Blood 2010, 116:6092-6100.

31. Hagel M, George EL, Kim A, Tamimi R, Opitz SL, Turner CE, Imamoto A, Thomas SM: The adaptor protein paxillin is essential for normal 
development in the mouse and is a critical transducer of fibronectin signaling. Mol Cell Biol 2002, 22:901-915.

32. Feral CC, Rose DM, Han J, Fox N, Silverman GJ, Kaushansky K, Ginsberg MH: Blocking the alpha 4 integrin-paxillin interaction selectively impairs mononuclear leukocyte recruitment to an inflammatory site. J Clin Invest 2006, 116:715-723.

33. Dorsam RT, Kim S, Jin J, Kunapuli SP: Coordinated signaling through both G12/13 and G (i) pathways is sufficient to activate GPIIb/Illa in human platelets. J Biol Chem 2002, 277:47588-47595.

34. Nieswandt B, Schulte V, Zywietz A, Gratacap MP, Offermanns S: Costimulation of Gi- and G12/G13-mediated signaling pathways induces integrin alpha llbbeta 3 activation in platelets. J Biol Chem 2002, 277:39493-39498.

35. Gupta S, Braun A, Morowski M, Premsler T, Bender M, Nagy Z, Sickmann A, Hermanns HM, Bosl M, Nieswandt B: CLP36 Is a Negative Regulator of Glycoprotein VI Signaling in Platelets. Circ Res 2012, 111:1410-1420.

36. Kim-Kaneyama JR, Miyauchi A, Lei XF, Arita S, Mino T, Takeda N, Kou K, Eto K, Yoshida T, Miyazaki T, et al: Identification of Hic-5 as a novel regulatory factor for integrin alphallbbeta3 activation and platelet aggregation in mice. J Thromb Haemost 2012, 10:1867-1874.

37. Chew V, Lam KP: Leupaxin negatively regulates $B$ cell receptor signaling. J Biol Chem 2007, 282:27181-27191

doi:10.1186/1477-9560-12-1

Cite this article as: Sakata et al:: Paxillin is an intrinsic negative regulator of platelet activation in mice. Thrombosis Journal 2014 12:1.

\section{Submit your next manuscript to BioMed Central and take full advantage of:}

- Convenient online submission

- Thorough peer review

- No space constraints or color figure charges

- Immediate publication on acceptance

- Inclusion in PubMed, CAS, Scopus and Google Scholar

- Research which is freely available for redistribution 